\title{
The fecal microbiome directly drives immune activation in HIV infection
}

\author{
Camilla Tincati, Giuseppe Ancona, Giulia Marchetti \\ Clinic of Infectious Diseases, Department of Health Sciences, ASST Santi Paolo e Carlo, Presidio San Paolo, University of Milan, Milan, Italy \\ Correspondence to: Camilla Tincati, MD, PhD. Clinic of Infectious Diseases, Department of Health Sciences, San Paolo Hospital, University of Milan, \\ Via di Rudini’ 8, 20142, Milan, Italy. Email: camilla.tincati@unimi.it. \\ Comment on: Neff CP, Krueger O, Xiong K, et al. Fecal Microbiota Composition Drives Immune Activation in HIV-infected Individuals. \\ EBioMedicine 2018;30:192-202.
}

Submitted Sep 20, 2018. Accepted for publication Sep 29, 2018.

doi: 10.21037/atm.2018.09.66

View this article at: http://dx.doi.org/10.21037/atm.2018.09.66

Immune activation is a hallmark of human immunodeficiency virus (HIV) infection: it causes progression to the acquired immunodeficiency syndrome (AIDS), impaired CD4+ T-cell reconstitution on suppressive combination antiretroviral therapy (cART) and contributes to non-infectious comorbidities (1-6). Understanding the mechanisms whereby immune activation arises is thus crucial for the elaboration of novel therapeutic interventions in the setting of HIV infection.

Microbial translocation, i.e., the passage of bacteria and microbial bioproducts through the gastrointestinal (GI) tract to the peripheral circulation, was described as a possible cause of immune activation over a decade ago $(7,8)$. In the attempt to shed light on the mechanisms whereby microbial translocation arises in the context of HIV infection, research studies have proved the existence of anatomical and immune defects within the GI tract of infected individuals (9-14) as well as profound modifications of the intestinal microbiota (15-19). In particular, HIVrelated dysbiosis features the depletion of Bacteroides and the enrichment for Proteobacteria, which, respectively, limit and promote inflammation $(15,17)$, thus implying that precise compositional changes of the microbiota contribute to immune activation in HIV. Consistently, studies have demonstrated that single microbial components are able to induce the activation of adaptive and innate immune cells in vitro $(20,21)$ and that $\mathrm{HIV}$-related dysbiosis correlates with markers of immune activation and disease progression $(15,22,23)$. However, whether the fecal microbiome in $\mathrm{HIV}$-infected individuals directly drives peripheral immune activation is currently unknown.

In the paper, "Fecal Microbiota Composition Drives Immune Activation in HIV-infected Individuals" by Neff et al. published in EBioMedicine, the authors take an interesting approach to the research question by performing in vitro stimulation assays with fecal bacteria communities (FBCs) (24) and human cells. Stool and peripheral blood samples were collected from HIV-infected and uninfected individuals: the former was used to develop a novel method for purification of intact microbial cells; the latter were employed to isolate peripheral blood mononuclear cells (PBMCs), monocytes and CD4+ T-cells for a comprehensive study of the inflammatory properties of $\mathrm{HIV}$-associated enteric microbiota.

In contrast to previous findings, HIV infection did not result the predominant factor linked to the reported differences in the composition fecal microbiome: the predominance of Prevotella-rich/Bacteroides-poor microbiomes was in fact observed in men who have sex with men (MSM) and corroborate previous research on the clustering of the microbiome composition according to sexual behaviour (25). Of note, however, they detected a greater production of pro-inflammatory cytokines in monocyte cultures and the selective activation of peripheral T-cells following culture stimulation with FBCs from HIV-infected MSM and not uninfected MSM, thus demonstrating the pathogenic role of the gut microbiome in HIV disease. From a mechanistic standpoint, tumour necrosis factor (TNF)- $\alpha$ and toll-like receptor (TLR)-2 resulted strong mediators of adaptive immunity activation. 
The present paper is noteworthy for several reasons. First, the authors were able to purify FBCs which maintained similar microbiota community membership to whole stool, thus recapitulating in vitro the interactions among different components of the microbiome. Second, the finding of higher levels of innate and adaptive immune activation in HIV-infected MSM following FBCs stimulation paves the way to new research questions, such as what is the clinical risk of disease progression/noninfectious comorbidities in this specific patient population. Finally, by demonstrating the mechanisms by which the fecal microbiota induces immune activation, this paper highlights possible targets for new therapeutic interventions in the setting of HIV infection.

\section{Acknowledgements}

Funding: This work was supported by the Italian Ministry of Health, Regione Lombardia, grant "Giovani Ricercatori" (number GR-2009-1592029; PI: G Marchetti) and grant "Ricerca Finalizzata-Progetti di Rete" (number NET-201302355333-3; PI: G Marchetti).

\section{Footnote}

Conflicts of Interest: The authors have no conflicts of interest to declare.

\section{References}

1. Liu Z, Hultin LE, Cumberland WG, et al. Elevated relative fluorescence intensity of CD38 antigen expression on CD8+ T cells is a marker of poor prognosis in HIV infection: results of 6 years of follow-up. Cytometry 1996;26:1-7.

2. Giorgi JV, Hultin LE, McKeating JA, et al. Shorter survival in advanced human immunodeficiency virus type 1 infection is more closely associated with $\mathrm{T}$ lymphocyte activation than with plasma virus burden or virus chemokine coreceptor usage. J Infect Dis 1999;179:859-70.

3. Hunt PW, Martin JN, Sinclair E, et al. T cell activation is associated with lower $\mathrm{CD} 4+\mathrm{T}$ cell gains in human immunodeficiency virus-infected patients with sustained viral suppression during antiretroviral therapy. J Infect Dis 2003;187:1534-43.

4. Deeks SG, Kitchen CM, Liu L, et al. Immune activation set point during early HIV infection predicts subsequent
CD4+ T-cell changes independent of viral load. Blood 2004;104:942-7.

5. Marchetti G, Gori A, Casabianca A, et al. Comparative analysis of $\mathrm{T}$-cell turnover and homeostatic parameters in HIV-infected patients with discordant immune-virological responses to HAART. AIDS 2006;20:1727-36.

6. Duffau P, Ozanne A, Bonnet F, et al. Multimorbidity, age-related comorbidities and mortality: association of activation, senescence and inflammation markers in HIV adults. AIDS 2018;32:1651-60.

7. Brenchley JM, Price DA, Schacker TW, et al. Microbial translocation is a cause of systemic immune activation in chronic HIV infection. Nat Med 2006;12:1365-71.

8. Marchetti G, Bellistrì GM, Borghi E, et al. Microbial translocation is associated with sustained failure in CD4+ T-cell reconstitution in HIV-infected patients on long-term highly active antiretroviral therapy. AIDS 2008;22:2035-8.

9. Brenchley JM, Schacker TW, Ruff LE, et al. CD4+ T cell depletion during all stages of HIV disease occurs predominantly in the gastrointestinal tract. J Exp Med 2004;200:749-59.

10. Mehandru S, Poles MA, Tenner-Racz K, et al. Mechanisms of gastrointestinal CD4+ T-cell depletion during acute and early human immunodeficiency virus type 1 infection. J Virol 2007;81:599-612.

11. Nazli A, Chan O, Dobson-Belaire WN, et al. Exposure to HIV-1 directly impairs mucosal epithelial barrier integrity allowing microbial translocation. PLoS Pathog 2010;6:e1000852.

12. Chung CY, Alden SL, Funderburg NT, et al. Progressive proximal-to-distal reduction in expression of the tight junction complex in colonic epithelium of virally-suppressed HIV+ individuals. PLoS Pathog 2014;10:e1004198.

13. Somsouk M, Estes JD, Deleage C, et al. Gut epithelial barrier and systemic inflammation during chronic HIV infection. AIDS 2015;29:43-51.

14. Tincati C, Merlini E, Braidotti $P$, et al. Impaired gut junctional complexes feature late-treated individuals with suboptimal CD4+ T-cell recovery upon virologically suppressive combination antiretroviral therapy. AIDS 2016;30:991-1003.

15. Vujkovic-Cvijin I, Dunham RM, Iwai S, et al. Dysbiosis of the gut microbiota is associated with HIV disease progression and tryptophan catabolism. Sci Transl Med 2013;5:193ra91.

16. Gori A, Tincati C, Rizzardini G, et al. Early impairment of 
gut function and gut flora supporting a role for alteration of gastrointestinal mucosa in human immunodeficiency virus pathogenesis. J Clin Microbiol 2008;46:757-8.

17. Dillon SM, Lee EJ, Kotter CV, et al. An altered intestinal mucosal microbiome in HIV-1 infection is associated with mucosal and systemic immune activation and endotoxemia. Mucosal Immunol 2014;7:983-94.

18. Vázquez-Castellanos JF, Serrano-Villar S, Latorre A, et al. Altered metabolism of gut microbiota contributes to chronic immune activation in $\mathrm{HIV}$-infected individuals. Mucosal Immunol 2015;8:760-72.

19. Lozupone CA, Li M, Campbell TB, et al. Alterations in the gut microbiota associated with HIV-1 infection. Cell Host Microbe 2013;14:329-39.

20. Tincati C, Bellistrì GM, Ancona G, et al. Role of in vitro stimulation with lipopolysaccharide on T-cell activation in $\mathrm{HIV}$-infected antiretroviral-treated patients. Clin Dev Immunol 2012;2012:935425.

Cite this article as: Tincati C, Ancona G, Marchetti G. The fecal microbiome directly drives immune activation in HIV infection. Ann Transl Med 2018;6(Suppl 1):S45. doi: 10.21037/ atm.2018.09.66
21. Merlini E, Tincati C, Biasin M, et al. Stimulation of PBMC and Monocyte-Derived Macrophages. Front Immunol 2016;7:614.

22. Ellis CL, Ma ZM, Mann SK, et al. Molecular characterization of stool microbiota in HIV-infected subjects by panbacterial and order-level $16 \mathrm{~S}$ ribosomal DNA (rDNA) quantification and correlations with immune activation. J Acquir Immune Defic Syndr 2011;57:363-70.

23. Vujkovic-Cvijin I, Swainson LA, Chu SN, et al. GutResident Lactobacillus Abundance Associates with IDO1 Inhibition and Th17 Dynamics in SIV-Infected Macaques. Cell Rep 2015;13:1589-97.

24. Neff CP, Krueger O, Xiong K, et al. Fecal Microbiota Composition Drives Immune Activation in HIV-infected Individuals. EBioMedicine 2018;30:192-202.

25. Noguera-Julian M, Rocafort M, Guillén Y, et al. Gut Microbiota Linked to Sexual Preference and HIV Infection. EBioMedicine 2016;5:135-46. 